\title{
Negative long wavelength dielectric constant in semiconductor QWs
}

\author{
S. Hessami Pilehrood ${ }^{1}$, Feng $\mathrm{Gao}^{1}$, Zhongshui $\mathrm{Ma}^{2}$, Wen $\mathrm{Xu}^{3}$, and Chao Zhang*1 \\ ${ }^{1}$ School of Engineering Physics, University of Wollongong, New South Wales, 2522, Australia \\ ${ }^{2}$ State Key Laboratory for Mesoscopic Physics and Department of Physics, Peking University, Beijing \\ 100871, China \\ ${ }^{3}$ Department of Theoretical Physics, Research School of Physical Sciences and Engineering, Australian \\ National University, Canberra, ACT 0200, Australia
}

Received 30 July 2006, revised 15 August 2006, accepted 15 August 2006

Published online 7 February 2007

PACS 71.45.Gm, 71.70.Ej, 78.67.De

In this work we present an analytical and numerical result of the dielectric function of electronic systems with Rashba spin-orbit interaction. By solving the quantum equation of motion for the electron density matrix, we obtained the dielectric function in the mean-field approximation. It is shown that when the momentum transfer $q$ for an electronic transition is smaller than a critical value characterized by the spinorbit interaction, the static dielectric function increases with $q$, indicating on-set of a new collective state.

(c) 2007 WILEY-VCH Verlag GmbH \& Co. KGaA, Weinheim

1 Introduction In spintronics, the central task is to find an effective way to manipulate and control the spin polarization and spin transport [1]. In two dimensional semiconductors, the spin-orbit interaction is mainly due to the Rashba spin-orbit interaction (SOI). This Rashba term not only leads to the level splitting, but also produces a transverse force in the direction perpendicular to the applied electric field. This transverse force results in the charge and spin Hall current under zero magnetic field $[2,3]$. The Coulomb interaction between electrons can modify and, to some extent, enhance the spin-orbit interaction and spin splitting. The spintronic systems and devices can be realized on the basis of diluted magnetic semiconductors and narrow-gap semiconductor nanostructures. In typical spintronic systems, electron states with opposite spin acquire different phase factors during their propagation in the presence of Rashba spin-orbit coupling [4,5]. We have shown recently [6] that two-colour plasmon excitation can be achieved in a spintronic double layer system and the excitation characteristics can be tuned by the SOI. In this work, we present an analytical result for the static dielectric function for a semiconductor quantum well with Rashba coupling. Our result indicates a new collective state when the momentum exchange is smaller than a critical value $q_{c}$.

We consider a quantum well where electrons move freely on the $x-y$ plane. The single electron Hamiltonian, the eigenfunctions, and the energy eigenvalues can be expressed respectively as

$$
\begin{aligned}
& H=\frac{1}{2 m^{*}}\left(p_{x}^{2}+p_{y}^{2}\right)+\frac{\lambda}{\hbar}\left(\sigma_{y} p_{x}-\sigma_{x} p_{y}\right), \\
& \psi\left(k_{x}, k_{y}\right)=u_{\mathbf{k}}(x, y) \xi_{\mathbf{k} \alpha}=\frac{1}{(2 \pi)^{2}} \exp \left(i k_{x} x+i k_{y} y\right) \frac{1}{\sqrt{2}}\left(\begin{array}{c}
1 \\
-\alpha\left(k_{y}-i k_{x}\right) / k
\end{array}\right),
\end{aligned}
$$

* Corresponding author: e-mail: czhang@uow.edu.au, Phone: +61 242213458, Fax: +61 242213238 
and $E_{\alpha}^{(0)}=E_{k, \alpha}=\frac{\hbar^{2}}{2 m^{*}} k^{2}+\alpha \lambda k$, where $m^{*}$ and $\sigma_{x}\left(\sigma_{y}\right)$ are the electron effective mass and the $x$ (y) component of the spin Pauli matrices respectively, $\lambda$ represents the strength of the spin-orbit coupling, $\alpha= \pm 1$ corresponds to the SOI induced branches, and $k=\sqrt{k_{x}^{2}+k_{y}^{2}}$. The single particle states, given by the Eq. (2), provide a convenient basis in constructing the field operators and the Hamiltonian for the many-electron system in the presence of electron-impurity interaction, to obtain the single electron density matrix of the system and the electronic polarization. Using the eigenstates represented by the Eq. (2), the electron field operator can be constructed as $\widehat{\Psi}(x, y)=\sum_{k, \alpha} \widehat{a}_{\mathbf{k} \alpha} u_{\mathbf{k}}(x, y) \xi_{\mathbf{k} \alpha}$. The second-quantized form of the Hamiltonian will be obtained according to $H=H_{0}+H_{e e}+H_{i m}$, where $H_{0}, H_{e e}$ and $H_{i m}$ are single particle energy, electron-electron interaction and electron interaction with impurities [2].

2 Density fluctuation and the dielectric functions We now calculate the dielectric function of the system. The single-electron density matrix element between states $\langle k, t|$ and $\mid k+p, t>$ is defined as $F_{\sigma \sigma^{\prime}}(\mathbf{k}+\mathbf{p}, \mathbf{k}, t)=<\widehat{a}_{\mathbf{k}, \sigma^{\prime}}^{\dagger}(t) \widehat{a}_{\mathbf{k}+\mathbf{p}, \sigma}(t)>$. Here we choose to use spin quantum number $\sigma$ instead of the Rashba band index $\alpha$. The electron density fluctuation is given as $n(q, t)=\sum_{\mathbf{k} \sigma} F_{\sigma \sigma^{\prime}}(\mathbf{k}+\mathbf{p}, \mathbf{k}, t)$. By solving the equation of motion $i \hbar \partial F_{\sigma \sigma^{\prime}}(\mathbf{k}+\mathbf{p}, \mathbf{k}, t) / \partial t=[F, H]$ in the static limit, we found the density fluctuation due to electron-impurity interaction,

$$
n(\mathbf{q})=\frac{1}{2} e^{2} Z \frac{2 V(|\mathbf{q}|) Q(\mathbf{q}, 0)}{\epsilon(\mathbf{q}, 0)} \sum_{i} \exp \left(-i \mathbf{q} \cdot \mathbf{R}_{\mathbf{i}}\right)
$$

where $V(q)=2 \pi e^{2} / q$ and $\mathbf{R}_{i}$ is the position of $i^{t h}$ impurities. In the above equation $Q(\mathbf{q}, 0)$ and $D(\mathbf{q}, 0)$ are the electronic polarizability and the dielectric function in the static limit, given as

$$
Q(\mathbf{q}, \omega=0, \lambda)=\sum_{\mathbf{k}} \frac{\Delta\left[\Delta^{2}-\lambda^{2}\left(4 k^{2}+q^{2}+4 \mathbf{k} \cdot \mathbf{q}\right)\right] \sum_{\alpha}\left(f_{\mathbf{k}+\mathbf{q}, \alpha}-f_{\mathbf{k}, \alpha}\right)}{\left[\Delta^{2}-\lambda^{2}\left(q^{2}+2 \mathbf{k} \cdot \mathbf{q}\right)\right]^{2}-(2 \lambda k \Delta)^{2}},
$$

and $D(\mathbf{q}, \omega=0)=1-V(|\mathbf{q}|) Q(\mathbf{q}, \omega=0)$. Here $\Delta=\hbar^{2}(\mathbf{k}+\mathbf{q})^{2} / 2 m^{*}-\hbar^{2} k^{2} / 2 m^{*}$ and $f_{\mathbf{k}, \alpha}$ is the Fermi-Dirac distribution function.

3 Results and discussions The integration over the k-space in Eq. (4) can be carried out analytically. After rather long algebraic manipulations, we found,

$$
\begin{aligned}
D(\mathbf{q}, 0, \lambda)= & +2 \frac{r_{s}}{q}+\frac{r_{s}}{2 q^{2}}\left(1-\delta_{\lambda, 0}\right)\left\{\sqrt { ( q ^ { 2 } - \lambda ^ { 2 } ) } \left[2 \Theta(\lambda-z) \ln \left(w /\left(\sqrt{q^{2}+\lambda^{2}-4}+q\right)\right)\right.\right. \\
& +\Theta\left(5 \lambda+2 q-\sqrt{20-q^{2}}\right)\left(\ln (w-2 \lambda)-\ln \left(q+\sqrt{q^{2}-(w-2 \lambda)^{2}}\right)\right) \\
& \left.+2 \Theta\left(2 \lambda+q-\sqrt{8-q^{2}}\right) \ln \left(\frac{\sqrt{q^{2}-\lambda^{2}}+\sqrt{q^{2}-4+2 \lambda w}}{\sqrt{\left|\lambda^{2}-4+2 \lambda w\right|}}\right)\right] \Theta(q-\lambda) \\
& -\sqrt{\left(\lambda^{2}-q^{2}\right)}\left[\frac{3 \pi}{2}+\arcsin \left(\frac{q}{w+2 \lambda}\right)+\Theta(\lambda-z)\left(\arcsin \left(\frac{q}{w}\right)-\frac{\pi}{2}\right)\right. \\
& +\operatorname{sgn}\left(5 \lambda-\sqrt{20-q^{2}}\right) \Theta\left(\left|5 \lambda-\sqrt{20-q^{2}}\right|-2 q\right)\left(\arcsin \left(\frac{q}{|w-2 \lambda|}\right)-\frac{\pi}{2}\right) \\
& \left.\left.-2 \Theta(\xi) \arcsin \left(\frac{\sqrt{q^{2}-4+2 \lambda w}}{\sqrt{\lambda^{2}-4+2 \lambda w}}\right)\right] \Theta(\lambda-q)-2 \Theta(\xi) \sqrt{q^{2}-4+2 \lambda w}\right\} .
\end{aligned}
$$

where $w=\sqrt{4-\lambda^{2}}, z=\sqrt{4-q^{2}}, \xi=2 \lambda+q-\sqrt{8-q^{2}}$. In the above equation, all energies are in unit of $E_{F}(0)$, all momenta are in unit of $k_{F}(0)$ and $\lambda$ is in unit of $E_{F}(0) / k_{F}(0), r_{s}=m^{*} e^{2} / \hbar^{2} k_{F}(0)$, where $E_{F}(0)$ and $k_{F}(0)$ are the Fermi energy and momentum in the absence of spin-orbit coupling. $\Theta(x)$ 

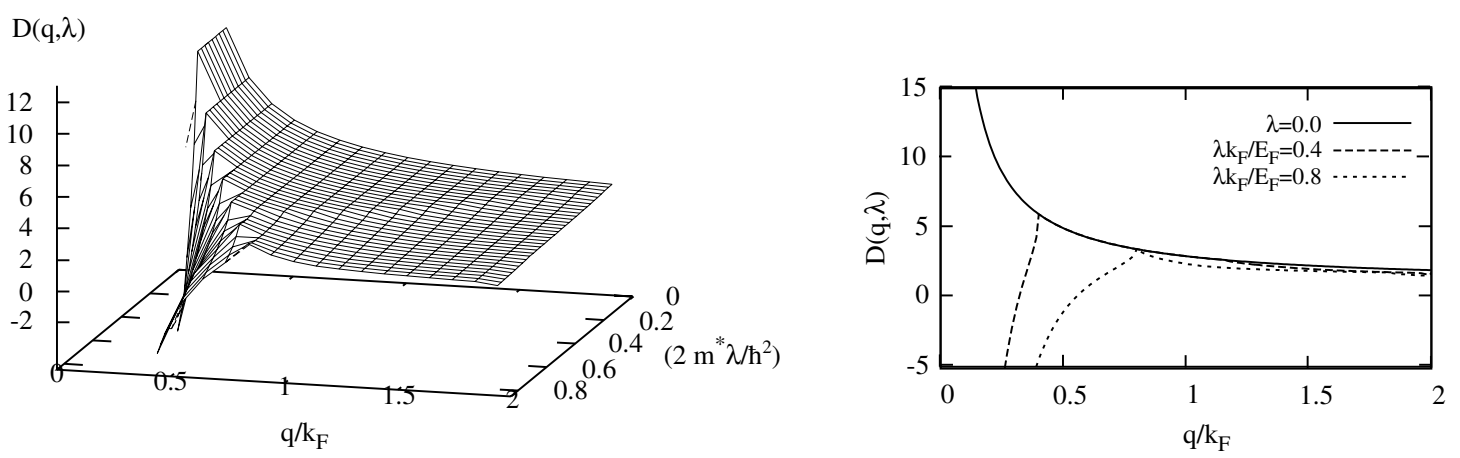

Fig. 1 (Left figure) The wave-vector and Rashba SOI dependent dielectric function of a 2DEG. (Right figure) Wave vector dependence of the dielectric function of a $2 \mathrm{DEG}$ with Rashba spin orbit coupling.

is the Heaviside step function. This equation will be useful in understanding the effects of the Rashba spin orbit coupling on the many body properties of an electronic system.

The exact result for the static dielectric function, the equation (5), has been illustrated in the Fig. 1. The left figure shows the dielectric function of the system as a function of the change in the electron wave vector $q$ and Rashba parameter $\lambda$. The right figure shows the dependence of the dielectric function on the wave vector changes for three different Rashba parameters. The solid line is the dielectric function without the spin orbit coupling. The dashed line and the dotted line show the dielectric function respectively for the normalized Rashba parameter $\lambda k_{F} / E_{F}$ equal to 0.4 and 0.8 . There are two completely distinctive regimes.

The $q>2 m^{*} \lambda / \hbar^{2}$ regime is not affected by the spin orbit coupling. According to [7] this can be due to the invariance of spatial wave function of electrons in different spin branches. Since the electrons with the same wave vector but in different splitting branches have antiparallel spins, the vertical inter-branch transitions are forbidden under spin-independent Coulomb interaction. The non-vertical transitions are weak and have negligible effects in this regime except for the case of very strong spin orbit couplings. In the other regime, where $q<2 m^{*} \lambda / \hbar^{2}$, the dramatic changes in the dielectric function may be attributed to the transitions between different Rashba branches. In the absence of SOI, the static dielectric function increases rapidly as $q$ approaches 0 . This rapid increase is mainly due to the vanishing transition energy $\Delta \rightarrow 0$ as $q \rightarrow 0$. In the presence of SOI, $\Delta \rightarrow 0$ at a slower rate. This leads to a less singular behavior for the intra-level transitions. Furthermore, there are two inter-level transitions which are nonsingular at $q=0$ and can contribute a negative term to the total dielectric function. As a result, the total dielectric function decreases with the wave-vector at small $q$.

Acknowledgements This work is supported in part by the Australian Research Council through a Linkage-International grant.

\section{References}

[1] S. A. Wolf, D. D. Awschalom, R. A. Buhrman, J. M. Daughton, S. von Molnar, M. L. Roukes, A. Y. Chtchelkanova, and D. M. Treger, Science 294, 1488 (2001).

[2] C. Zhang and Z. S. Ma, Phys. Rev. B 71, 121307 (2005).

[3] Jairo Sinova et al., Phys. Rev. Lett. 78, 1335 (2004).

[4] E. I. Rashba, Fiz. Tverd. Tela (Leningrad) 2, 1224 (1960), [Sov. Phys. Solid State 2, 1109 (1960)].

[5] D. Loss and D. P. DiVincenzo, Phys. Rev. A 57, 120 (1998).

[6] C. H. Yang et al., Appl. Phys. Lett. 88, 223102 (2006).

[7] X. F. Wang, Phys. Rev. B 72, 085317 (2005). 